\title{
The Effect of $U$ Atom Adsorption on the Structural, Electronic and Magnetic Properties of Single-Walled Carbon Nanotubes
}

\author{
YA NIE, ${ }^{1,2}$ MU LAN, ${ }^{1,2}$ XI ZHANG, ${ }^{1,2}$ DINGYU YANG,${ }^{4}$ \\ and GANG XIANG (10) $1,2,3,5$ \\ 1.-College of Physical Science and Technology, Sichuan University, Chengdu 610064, China. \\ 2.-Key Laboratory of High Energy Density Physics and Technology of Ministry of Education, \\ Sichuan University, Chengdu 610064, China. 3.-Key Laboratory of Radiation Physics and \\ Technology of Ministry of Education, Sichuan University, Chengdu 610064, China. 4.-College of \\ Optoelectronic Technology, Chengdu University of Information Technology, Chengdu 610225, \\ China. 5.—e-mail: gxiang@scu.edu.cn
}

We investigate the structural, electronic and magnetic properties of three types of single-walled carbon nanotubes (SWNTs, zigzag, armchair and chiral) with adsorption of a uranium (U) atom using density-functional theories. Structural analysis shows that the site on the top of the hexagonal ring is the most stable for the $U$ atom adsorption on all the three types of SWNTs. Upon adsorption, chemical bonds are formed between $\mathrm{C}$ atoms and the $\mathrm{U}$ adatom, and the latter acts as a donor, resulting in a change of energy bands and increases the Fermi level of SWNTs. Interestingly, upon absorption of nonmagnetic U element, all types of the SWNTs become magnetic, and the induced magnetization is mainly contributed by the U adatom's $6 d$ and $5 f$ orbitals, which may be useful for carbon-based spintronic applications.

Key words: Magnetism, U atom, carbon nanotubes, adsorption, spintronics

\section{INTRODUCTION}

Single-walled carbon nanotubes (SWNTs) have been studied extensively both theoretically and experimentally since they were discovered. ${ }^{1}$ Because of their excellent physical and chemical properties, ${ }^{2,3}$ SWNTs and SWNT-based materials have been proposed for many applications including SWNT-FETs (field effect transistors), ${ }^{4}$ reinforcement materials, ${ }^{5}$ electrodes of Li-ion batteries ${ }^{6}$ and sensors. ${ }^{7-13}$ Among all these applications, adsorption of molecules (or atoms) on SWNTs have drawn a lot of attention due to the large surface-to-volume ratio of SWNTs. The modifications of the physical and chemical properties, such as electrical conductivity ${ }^{14}$ tensile strain ${ }^{15}$ and solubility, ${ }^{16}$ by adsorption of molecules have been studied. The correlation between the structures and electronic properties in nanoscale materials was also studied using

(Received October 24, 2017; accepted June 26, 2018; published online July 6, 2018) synchrotron x-ray microdiffraction technology. ${ }^{17-22}$ However, tuning of the magnetic properties of SWNTs via adsorption of molecules or atoms is rarely reported. On the other hand, the removal of heavy metal ions by carbon nanotubes is also scientifically and technologically important. ${ }^{23}$ In this paper, we investigate the $U$ atom adsorption of SWNTs (SWNTs:U) and study the adsorption effect on the electronic and magnetic properties of SWNTs. Although both pure SWNTs and U element are nonmagnetic, surprisingly, we find that SWNTs:U exhibits magnetism, which may be used for SWNT-based spintronic applications.

\section{METHODS}

We have performed the spin polarized first-principles calculation based on density-functional theory (DFT). The Vienna ab initio simulation package (VASP) code $^{24}$ is used. The projector augmented wave (PAW) potential ${ }^{25}$ is employed to describe the core electrons, and the generalized gradient approximation (GGA) of Perdew-Burke-Ernzerhof 


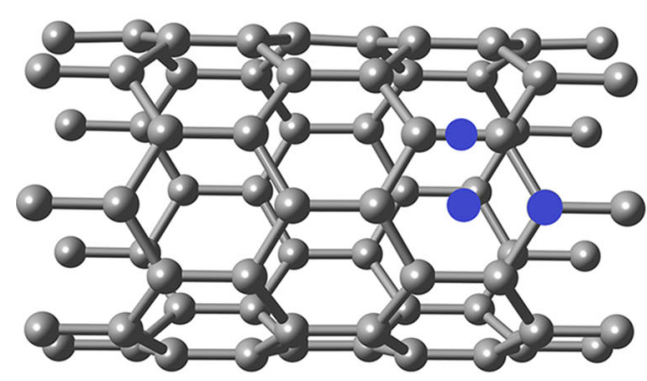

(a)

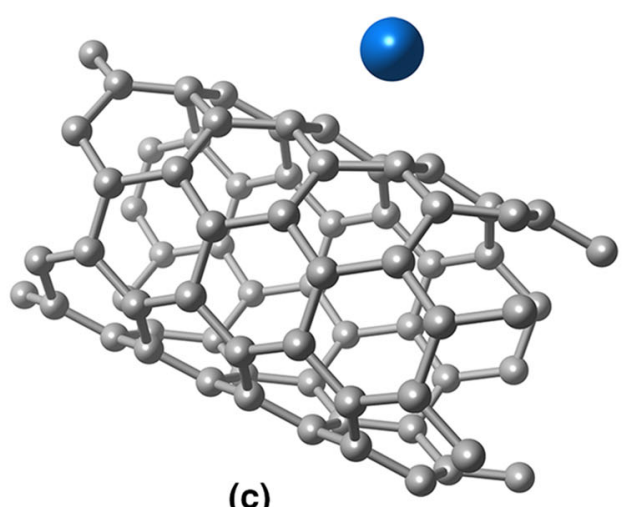

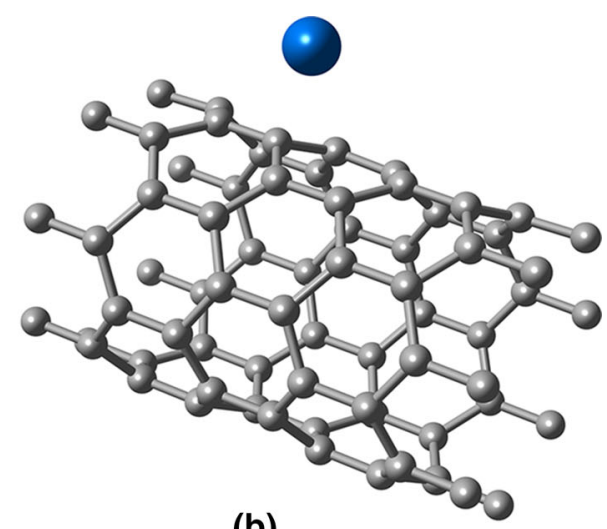

(b)

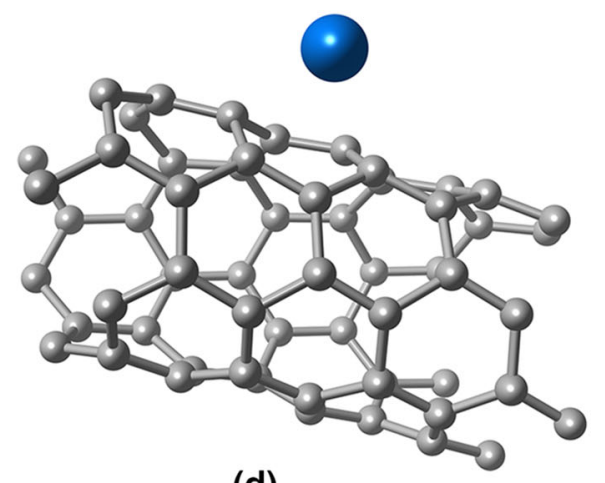

(d)

Fig. 1. Illustration of SWNTs with a $U$ adatom. The grey balls represent $C$ atoms and blue ones represent $U$. (a) The three possible sites for $U$ atom adsorption. The most stable site is the top of the hexagonal ring; (b) in Z-SWNT; (c) in A-SWNT; (d) in C-SWNT.

$(\mathrm{PBE})^{26}$ is adopted for exchange and correlation. The GGA + U approximation ${ }^{27}$ is taken into account to describe the localization of the $f$ electrons of $U$ element. With the Dudarev's approach, ${ }^{28}$ the single parameter $U_{\text {eff }}$ is chosen as $4 \mathrm{eV}$, which is very close to the value used by Durarev et al. ${ }^{29}$ and to some experimental findings. ${ }^{30,31}$ The kinetic energy cutoff of $520 \mathrm{eV}$ is used and the tolerance for electron convergence is set as $0.000001 \mathrm{eV}$ while the force is converged within $0.01 \mathrm{eV} / \AA$. Entire calculations, including relaxation calculations, static calculation and density of state (DOS) calculations, have been employed with a $(1 \times 1 \times 7) k$-mesh scheme. All atomic positions are optimized by minimization of the total energy and atomic forces, and collinear magnetic calculation is used to calculate magnetic moments.

Three types of SWNTs have been studied, including zigzag (7,0; Z-SWNT), armchair (4,4; A-SWNT) and chiral (5,2; C-SWNT). For Z-SWNTs, we investigate three possible sites for the $\mathrm{U}$ atom adsorption, including the hollow site (on the top of the hexagonal ring), bridge site (on the $\mathrm{C}-\mathrm{C}$ bond) and the top site (on top of one $\mathrm{C}$ atom), respectively, as shown in Fig. 1a. The structure of Z-SWNTs including $56 \mathrm{C}$
Table I. Average bond lengths $l$ and corresponding binding energies $\boldsymbol{E}_{\mathrm{b}}$ of the U-C bond in Z-SWNT:U, A-SWNT:U and C-SWNT:U

\begin{tabular}{|c|c|c|}
\hline U-C bond & $l(\AA)$ & $E_{b}(e V)$ \\
\hline Z-SWNT:U & 2.630 & 5.78 \\
\hline A-SWNT:U & 2.586 & 5.74 \\
\hline C-SWNT:U & 2.640 & 6.09 \\
\hline
\end{tabular}

atoms in each unit supercell was initially constructed maintaining a $\mathrm{C}-\mathrm{C}$ distance at $1.42 \AA$, and then optimized. After the optimization, one U atom was attached to the tube wall in each unit cell and the stability was investigated.

In order to discuss the stability, we calculate the binding energy $E_{\mathrm{b}}$ with the relation given by

$$
E_{\mathrm{b}}(\mathrm{U})=E(\mathrm{U})+E(\mathrm{SWNT})-E(\mathrm{SWNT}: \mathrm{U})
$$

where $E(\mathrm{U})$ is the energy of an isolated $\mathrm{U}$ atom, $E(\mathrm{SWNT})$ is the total energy of the pristine SWNT super-cell and $E(\mathrm{SWNT}: \mathrm{U})$ is the energy of the SWNT:U system. 

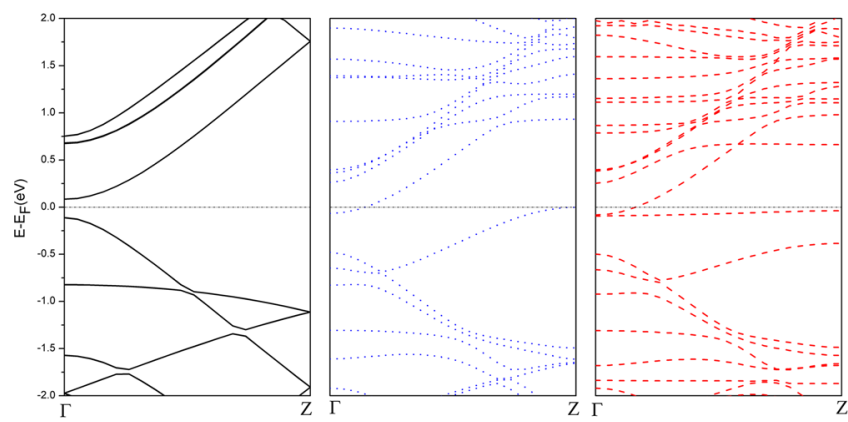

(a)
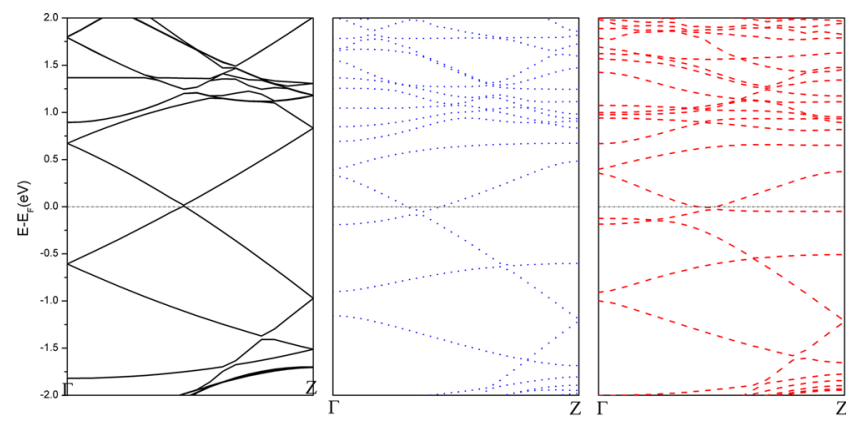

(b)
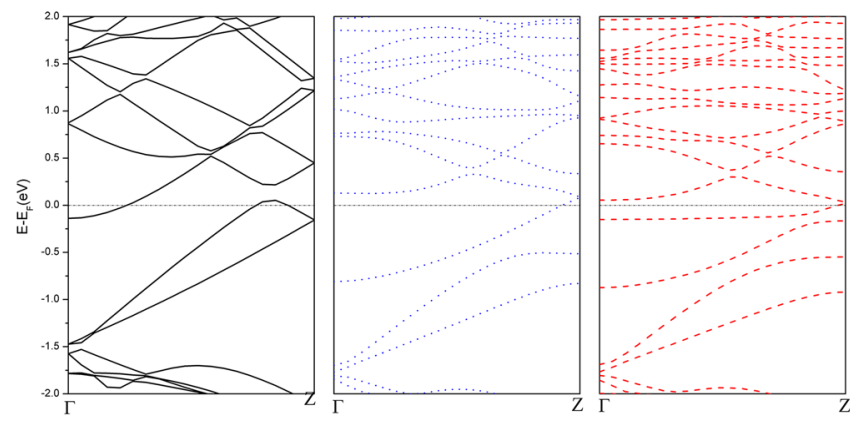

(c)

Fig. 2. Electronic band structures of (a) Z-SWNT (black line) and Z-SWNT:U (the blue line represents spin-up bands and the red line spin-down bands); (b) A-SWNT and A-SWNT:U; and (c) C-SWNT and C-SWNT:U (Color figure online).

Our calculation results show that the most stable site for $\mathrm{U}$ atom adsorption on Z-SWNTs is the hollow site, as shown in Fig. 1b. Similarly, for ASWNTs and C-SWNTs, the structures including 64 $\mathrm{C}$ atoms and $52 \mathrm{C}$ atoms in each unit cell are constructed and optimized, and the most stable sites for the adsorptions of $U$ atom are the hollow sites, too, as shown in Fig. 1c and d, respectively.

\section{RESULTS AND DISCUSSION}

Adsorbed on top of the hexagonal ring, the $\mathrm{U}$ adatom is about 2.4-2.8 $\AA$ away from its six nearest $\mathrm{C}$ atoms of the three types of SWNTs. The average bond lengths $(l)$ between $\mathrm{U}$ and its six nearest $\mathrm{C}$ atoms, and the corresponding binding energies are displayed in Table I. From Table I, one can see that C-SWNT:U has the largest binding energy with the largest average bond length, while A-SWNT:U has the smallest binding energy with the smallest average bond length. Z-SWNT:U lies in between.

The electronic structures of pure SWNT and SWNT:U systems are then investigated, as shown in Fig. 2. One can see that the adsorption of the U atom drives the band structures of SWNTs to redistribute, and makes contribution to the bands near the Fermi level. For instance, while Z-SWNT is a semiconductor, Z-SWNT:U becomes metallic, as shown in Fig. 2a. Interestingly, spin polarized bands appear and magnetism occurs after the U adsorption in all three types of the SWNTs, although all the pure SWNTs are nonmagnetic, as plotted in Fig. 2b and c.

In order to analyze the electronic and magnetic contributions, we plot the total density of states (TDOS) for the Z-SWNT:U systems in Fig. 3a. The partial DOSs (PDOS) of the U adatom and the six nearest $\mathrm{C}$ atoms near the Fermi level for all the three types of SWNTs are also presented in Fig. 3b, c and d. For Z-SWNT:U, one can see that the bands, which cross the Fermi level with both spin-up and spin-down parts, are evidently contributed by the U adatom's $7 s$ and $6 d$ electrons. The nonsymmetrical distribution of the $5 f$ orbitals is obvious, while nearly four electrons located in spin-down channel and spin-up channel stay almost empty. Then we can conclude that the magnetic moment stems from the charges of the $U$ adatom. Besides, in this metallic system, a charge transferring from the $U$ adatom to the SWNT occurs and thus the Fermi level of Z-SWNT moves up due to the reception of charge. Consequently, the $U$ adatom acts as a donor element. In fact, a very narrow band appears near the Fermi level in the spin-down part, which corresponds to the doping state of the $\mathrm{U}$ adatom. This U-doped system may be useful for spintronic applications. Similar outcomes are obtained for A-SWNT:U and C-SWNT:U, as plotted in Figs. 2 and 3.

Furthermore, analysis shows that there are several pairs of equal-energy $\mathrm{U} 6 d$ and $5 f$ peaks and $\mathrm{C}$ $2 p$ peaks located below the Fermi level. This 
The Effect of U Atom Adsorption on the Structural, Electronic and Magnetic Properties of Single-Walled Carbon Nanotubes
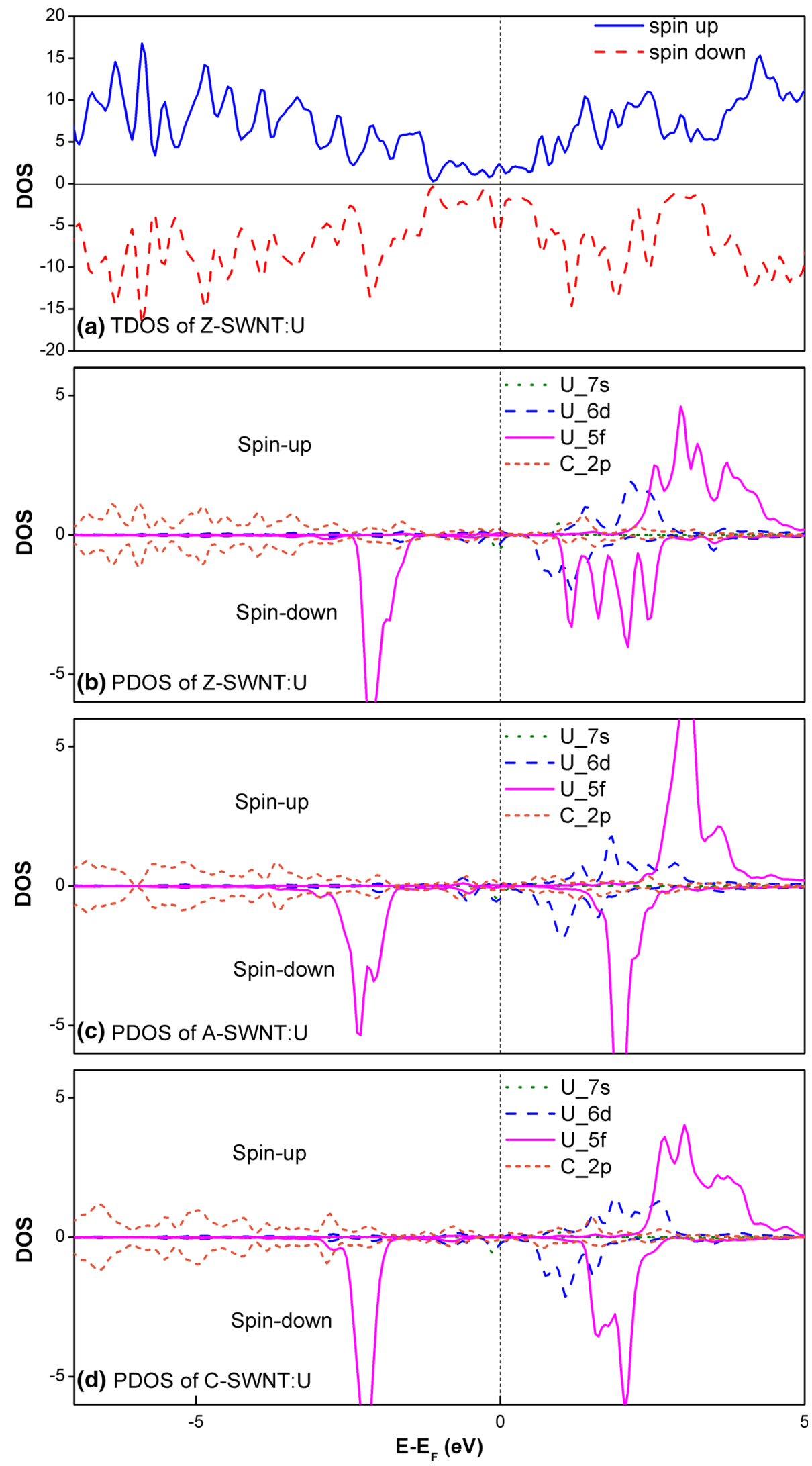

Fig. 3. (a) TDOS of Z-SWNT:U. The black dash line represents the Fermi level. The blue solid line stands for the spin-up charge and the red dashed line for spin-down. (b) PDOS of Z-SWNT:U; (c) PDOS of A-SWNT:U; (d) PDOS of C-SWNT:U. The green, blue and pink lines represent $7 s, 6 d, 5 f$ electrons of the $U$ atom, respectively. The orange lines represent $2 p$ electrons of the six $C$ atoms near $U$ (Color figure online). 


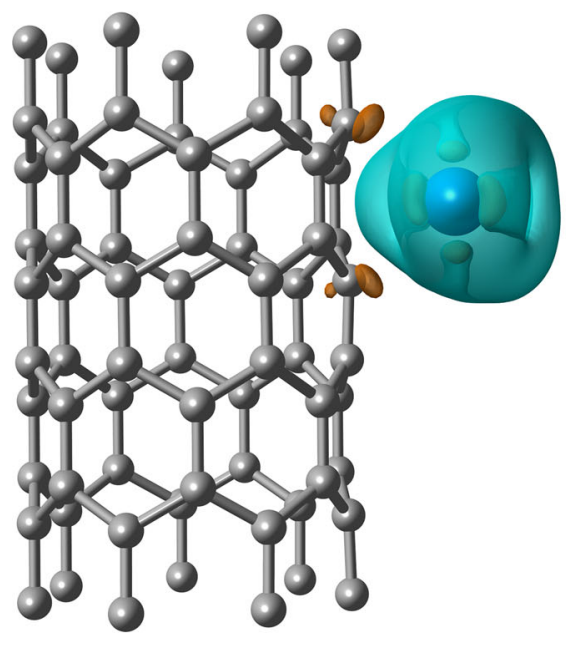

(a)

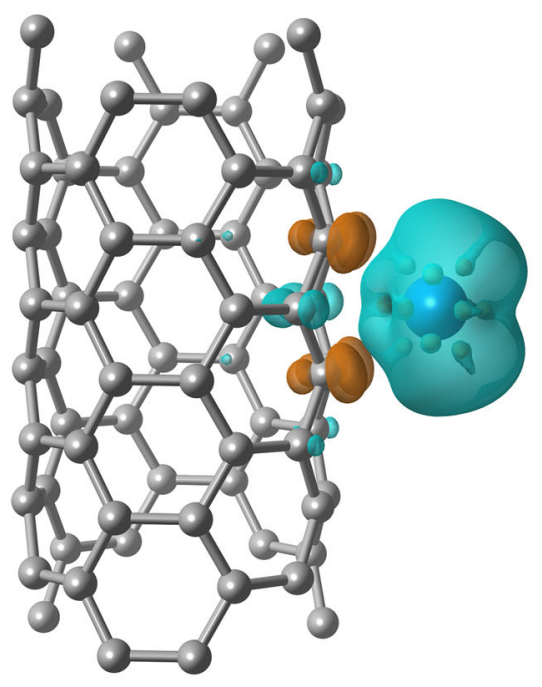

(b)

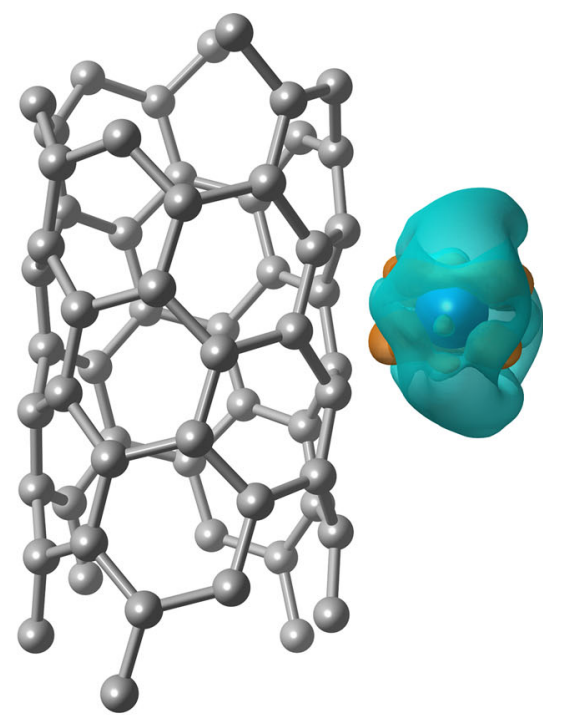

(c)

Fig. 4. Magnetization density of (a) Z-SWNT:U, (b) A-SWNT:U, and (c) C-SWNT:U. The orange (light blue) isosurface represents the spin-up (down) charges of $0.002 \mathrm{eV} / \AA^{3}$ (Color figure online).

Table II. The magnetic moments of $U$ atoms and total magnetic moments of corresponding supercells in Z-, A- and C-SWNT:U systems

\begin{tabular}{lcc}
\hline & Magnetic moment of $\mathbf{U}(\mu \mathbf{B})$ & Magnetic moment of supercell $(\mu \mathbf{B})$ \\
\cline { 2 - 3 } Z-SWNT:U & -3.85 & -3.92 \\
A-SWNT:U & -3.70 & -3.81 \\
C-SWNT:U & -3.90 & -4.03 \\
\hline
\end{tabular}

indicates that the hybridization of $\mathrm{U} 6 d$ and $5 f$ orbital and $\mathrm{C} 2 p$ orbital results in the adsorption of the U atom on Z-SWNTs by chemical bonds, not only by physical interaction. However, the contributions to the magnetic moment from $7 s$ orbitals are too little to be important because the $7 s$ shells are filled. Similar results are found for A-SWNT:U and CSWNT:U.

The magnetization density of the Z-SWNT:U system's ground state is shown in Fig. 4a, where the $U$ adatom shows a magnetic moment of $-3.85 \mu \mathrm{B}$ ( $\mu \mathrm{B}$ for a Bohr magneton. As a result, the $\mathrm{U}$ adatom has a spin-down magnetic moment, and induces small spin-up magnetic moments on the nearest two $\mathrm{C}$ atoms. It shows that although both pure SWNTs and the U element are nonmagnetic, the adsorption causes the magnetism in the SWNT:U system. Since magnetic materials can be easily and remotely collected by electromagnetic ways, the SWNT:U systems may find potential application in dealing with nuclear waste in nuclear power stations. The magnetization densities of ASWNT:U and C-SWNT:U are also plotted in Fig. 4b and c. The A-SWNT:U has the characteristics similar to Z-SWNT:U, but the inductive magnetic moments of $\mathrm{C}$ atoms are larger than those of $\mathrm{Z}$ SWNT:U. In Fig. 4b, we can see that the U atom has a spin-down magnetic moment and the nearest four $\mathrm{C}$ atoms possess spin-up magnetic moments; in addition, the farther $\mathrm{C}$ atoms possess spin-down magnetic moments. However, for the same minimum value of isosurface, C-SWNT:U induces negligible magnetic moments on $\mathrm{C}$ atoms near it.

For convenient comparisons, we plot the magnetic moments of $U$ adatoms in these three types SWNTs and total magnetic moments of corresponding supercells in Table II. As shown in Table II, the total magnetic moment for each type SWNT is mainly contributed by the U adatom's electrons.

\section{CONCLUSIONS}

In this work, the first-principles calculations are implemented to explore the structural, electronic and magnetic properties of U-adsorbed SWNTs. The stability of the adsorption is investigated and the top of the hexagonal ring is found to be the most stable site for SWNTs. The absorbed U atom acts as 
a donor and the adsorption of $\mathrm{U}$ atoms can effectively change the electronic structures of SWNTs. The magnetism occurs upon $\mathrm{U}$ atom adsorption for all the three types of SWNTs, which is resulted from the charge redistribution due to the adsorption and mainly contributed by $\mathrm{U} 6 d$ and $5 f$ orbitals. The SWNT:U systems maybe useful for applications in carbon-based spintronics.

\section{ACKNOWLEDGEMENTS}

This work was supported by the National Key R\&D Program of China through Grant No. 2017YFB0405702 and National Natural Science Foundation of China through Grant No. 51671137.

\section{REFERENCES}

1. S. Iijima, Nature 354, 56 (1991).

2. M. Ouyang, J.L. Huang, and C.M. Lieber, Acc. Chem. Res. 35, 1018 (2002).

3. M.F.L. De Volder, S.H. Tawfick, R.H. Baughman, and A.J. Hart, Science 339, 535 (2013).

4. H.R. Byon and H.C. Choi, J. Am. Chem. Soc. 128, 2188 (2006).

5. S. Kumar, T.D. Dang, F.E. Arnold, A.R. Bhattacharyya, B.G. Min, X. Zhang, R.A. Vaia, C. Park, W.W. Adams, R.H. Hauge, R.E. Smalley, S. Ramesh, and P.A. Willis, Macromolecules 35, 9039 (2002).

6. Ch Ban, Zh Wu, D.T. Gillaspie, L. Chen, Y. Yan, J.L. Blackburn, and A.C. Dillon, Adv. Mater. 22, E145 (2010).

7. J. Kong, N.R. Franklin, C. Chou, M.G. Chapline, S. Peng, K. Cho, and H. Dai, Science 287, 622 (2000).

8. H. Chang, J.D. Lee, S.M. Lee, and Y.H. Lee, Appl. Phys. Lett. 79, 3863 (2001).

9. J. Kong, M.G. Chapline, and H. Dai, Adv. Mater. 13, 1384 (2001).

10. J.J. Davis, K.S. Coleman, B.R. Azamian, C.B. Bagshaw, and M.L.H. Green, Chem. Eur. J. 9, 3732 (2003).

11. A. Qureshi, W.P. Kang, J.L. Davidson, and Y. Gurbuz, Diam. Relat. Mater. 18, 1401 (2009).
12. S.J. Pearton, F. Ren, Y.L. Wang, B.H. Chu, K.H. Chen, C.Y. Chang, W. Lim, J. Lin, and D.P. Norton, Prog. Mater Sci. 55, 1 (2010).

13. B.H. Son, J.Y. Park, S. Lee, and Y.H. Ahn, Nanoscale 7, 15421 (2015).

14. U. Dettlaff-Weglikowska, V. Skakalova, R. Graupner, L. Ley, and S. Roth, Mater. Res. Soc. Symp. Proc. 772, 179 (2003).

15. L.G. Zhou and S.Q. Shi, Comput. Mater. Sci. 23, 166 (2002).

16. Q.W. Li, J. Zhang, H. Yan, M.S. He, and Z.F. Liu, Carbon 42, 287 (2004).

17. N. Tamura, A.A. MacDowell, R. Spolenak, B.C. Valek, J.C. Bravman, W.L. Brown, R.S. Celestre, H.A. Padmore, B.W. Batterman, and J.R. Patel, J. Synchrotron Radiat. 10, 137 (2003).

18. A.S. Budiman, G. Lee, M.J. Burek, D. Jang, S.M. Han, N. Tamura, M. Kunz, J.R. Greer, and T.Y. Tsui, Mater. Sci. Eng. A 538, 89 (2012).

19. C.Y. Khoo, H. Liu, W. Sasangka, R.I. Made, N. Tamura, M. Kunz, A.S. Budiman, C.V. Thompson, and C.L. Gan, J. Mater. Sci. 51, 1864 (2016).

20. I. Radchenko, S.K. Tippabhotla, N. Tamura et al., J. Electr. Mater. 45, 6222 (2016).

21. H.P.A. Ali, I. Radchenko, J. Zhao, L. Qing, and A.S. Budiman, J. Mater. Des. Appl. 1, 1 (2017). https://doi.org/10. $1177 / 1464420717695354$

22. H.-A.-S. Shin, B.-J. Kim, J.-H. Kim, S.-H. Hwang, A.S. Budiman, H.-Y. Son, K.-Y. Byun, N. Tamura, M. Kunz, D.I. Kim, and Y.-C. Joo, J. Electron. Mater. 41, 712 (2012).

23. V.K. Gupta, O. Moradi, I. Tyagi, S. Agarwal, H. Sadegh, R. Shahryari-Ghoshekandi, A.S.H. Makhlouf, M. Goodarzi, and A. Garshasbi, Crit. Rev. Environ. Sci. Technol. 46, 93 (2016).

24. G. Kresse and J. Furthmuller, Phys. Rev. B 54, 11169 (1996).

25. P.E. Blöchl, Phys. Rev. B 50, 17953 (1994).

26. J.P. Perdew, K. Burke, and M. Ernzerhof, Phys. Rev. Lett. 77, 3865 (1996)

27. V.I. Anisimov, J. Zaanen, and O.K. Andersen, Phys. Rev. B 44, 943 (1991).

28. S.L. Dudarev, G.A. Botton, S.Y. Savrasov, C.J. Humphreys, and A.P. Sutton, Phys. Rev. B 57, 1505 (1998).

29. S.L. Dudarev, D. NguyenManh, and A.P. Sutton, Philos. Mag. B 75, 613 (1997).

30. J. Schoenes, J. Chem. Soc. Faraday Trans. II 83, 1205 (1987).

31. A. Kotani and T. Yamazaki, Prog. Theor. Phys. Suppl. 108, 117 (1992). 